\title{
Pre- and Post-aortic Endovascular Interventions: What a Radiologist Needs to Know
}

\author{
Thorsten Bley and Justus Roos
}

\section{Learning Objectives}

- To discuss elements of vascular anatomy pertinent to endovascular aneurysm repair of the thoracic and abdominal aorta

- To understand common complications of postendovascular stent-graft placement

\subsection{Introduction}

Aortic aneurysmal disease of the thoracic and abdominal aorta is a potentially life-threatening disease and requires besides preventive measures early detection of the disease and if present for the patient-tailored interventional treatment. Per definition, an abdominal aortic aneurysm is present if the abdominal aorta exceeds 1.5 times the normal diameter of 2.5-3 cm [1]. Many AAA remain asymptomatic and are found incidentally on routine imaging. Among a variable number of risk factors, such as arterial hypertension, inherited diseases, connective tissue diseases, and age, smoking appears to be the most important risk factor. Since rupture of an AAA is related with a very high mortality, repair is recommended with a size exceeding more than $5.0-5.5 \mathrm{~cm}$, if a patient turns symptomatic or an interval annual growth rate of $>1 \mathrm{~cm}$ is detected [2]. Endovascular aneurysmal repair (EVAR) surpassed the method of open surgical repair since approximately a decade and became the method of choice to treat AAA [3]. Newest generations of EVAR devices allow to

\footnotetext{
T. Bley $(\varangle)$

Institut für Diagnostische und Interventionelle Radiologie, Universitätsklinikum Würzburg, Würzburg, Deutschland e-mail: bley_t@ukw.de

J. Roos

Radiologie und Nuklearmedizin, Institut für Radiologie und Nuklearmedizin, Luzerner Kantonsspital, Luzern, Switzerland e-mail: justus.roos@1uks.ch
}

exclude the vast majority of AAA, despite their sometimes challenging anatomical morphology. Pre-procedural imaging is pivotal and important for EVAR to adequately assess the aneurysm size and anatomical configuration and with this to choose an adequate device and access site [4]. Post-procedural complications are relatively frequent (e.g., endoleaks) and demand regular post-EVAR surveillance [5]. Different imaging modalities, such as conventional radiography (CR), ultrasound (US), magnetic resonance angiography (MRA), and computed tomography angiography (CTA), play a role in preand post-procedural EVAR assessment with their relative strengths and weaknesses. In this article we focus on CTA as being the workhorse imaging modality used in the vast majority of institutions due to its high accuracy for pre- and postprocedural imaging [6].

\section{Key Point}

- An abdominal aortic aneurysm is present if the abdominal aorta exceeds 1.5 times the normal diameter of $2.5-3 \mathrm{~cm}$. CT angiography is considered the workhorse imaging modality used in the vast majority of institutions due to its high accuracy for pre- and post-procedural imaging.

EVAR devices are basically bifurcated metallic stents with a mounted layer of nonporous graft material. Their modular design normally includes one aortoiliac limb with an ipsilateral long component and a contralateral short limb that is secondly joined with a uniiliac component [4]. Different design variants are available to help to circumvent anatomical challenges. In general, EVAR endografts are placed with attention to the proximal and distal landing zones, where proper sealing of the device to the native aorta is crucial to exclude blood flow into the aneurysmal sac, thereby resulting in lowered sac pressures and decreased risk of further expansion. In case of incomplete exclusion of 
the blood flow influx into the aneurysmal sac, the AAA remains pressurized. Different sources, so-called endoleaks, are causative for a persistent blood flow into the aneurysmal sac $[7,8]$.

Similar to the EVAR procedure, several pathologies of the thoracic aorta and the aortic arch such as thoracic aortic aneurysm (TAA), penetrating aortic ulcer (PAU), intramural hemorrhage (IMH), traumatic aortic rupture, and aortic dissection (AD) can be treated with the so-called thoracic endovascular aortic repair (TEVAR) [9]. Technical success of TEVAR reaches $<90 \%$ in studies with substantially lower rates of neurologic complications and mortality as compared to open surgery [10-13]. No sternotomy or thoracotomy is necessary for the TEVAR procedure. TEVAR devices may consist of a short section of bare metal stent for better conformity and alignment in the aortic arch in order to reduce the risk of endoleak [14].

\section{Anatomical considerations for EVAR}

optimal versus (suboptimal)

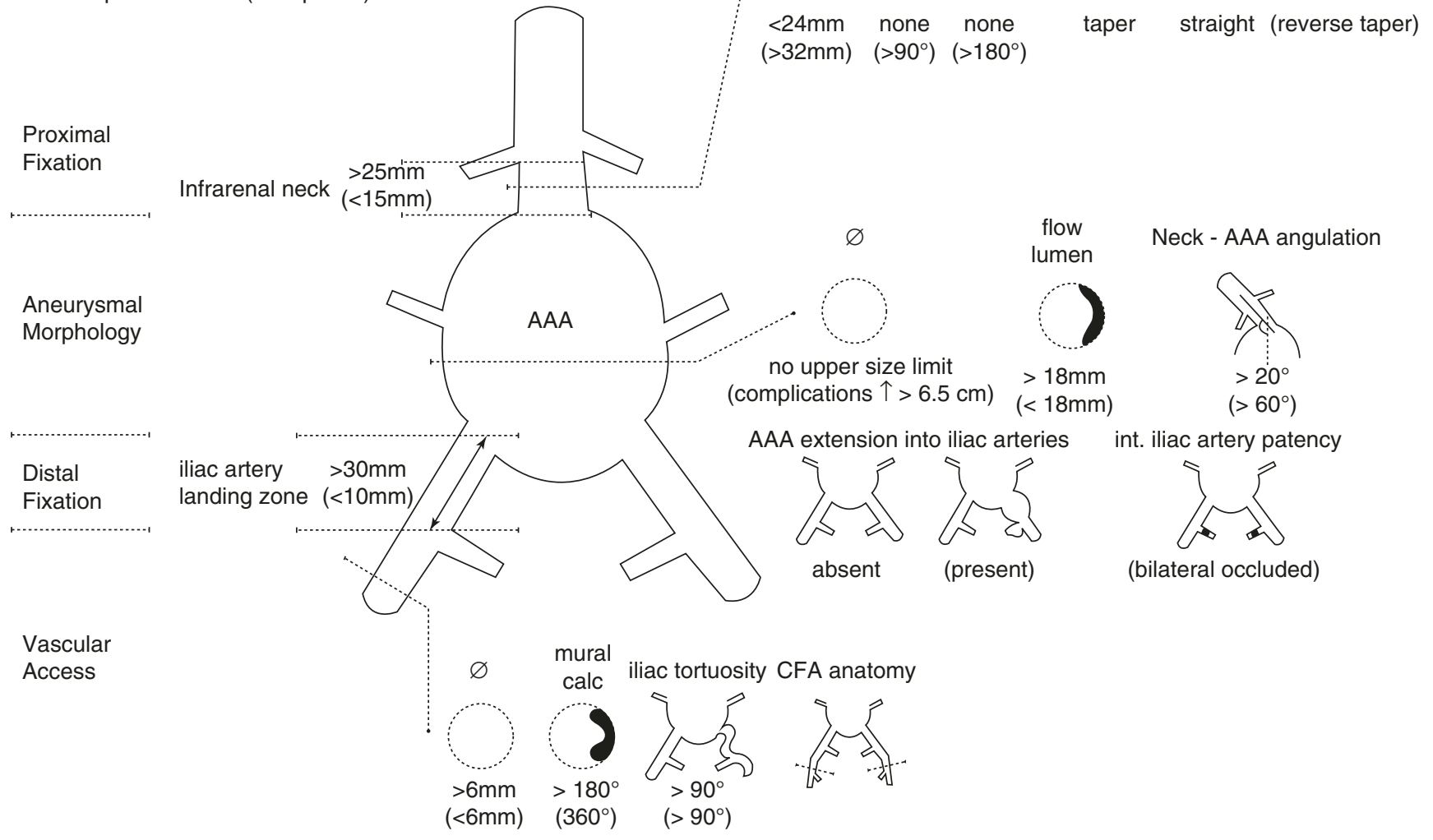

Fig. 19.1 Anatomical considerations to be addressed at pre-procedural imaging: Description of the proximal fixation site with infrarenal neck length, diameter, and configuration (mural thrombus, mural calcifications, shape). Description of the aneurysm morphology (saccular, fusiform), aneurysm size (largest diameter), aneurysm length, aneurysm flow lumen, aorta diameter at the level of the lowest renal artery and aortic bifurcation, and angle between supra-renal aorta and AAA. Description of the distal fixation site with the length of the iliac landing zone, iliac
Key Point

- Indications for endovascular aneurysmal repair of the abdominal aorta (EVAR) or the thoracic aorta (TEVAR) include besides aneurysmal disease, penetrating aortic ulcer (PAU), intramural hemorrhage (IMH), and (non-)traumatic aortic rupture or aortic dissection (AD).

\subsection{Pre-procedural Imaging}

Figure 19.1 summarizes a number of anatomic and morphologic parameters every pre-procedural CTA report needs to address, in order to reliably select the right EVAR device, to anticipate procedural challenges, and to plan for ancillary

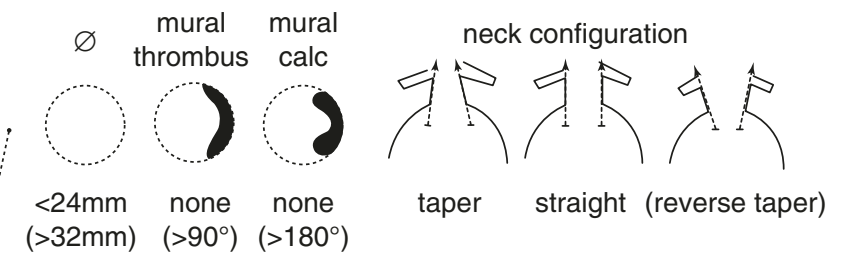

artery minimal diameter, iliac tortuosity and mural calcifications, extension of AAA into common iliac artery (CIA) aneurysms, and patency of internal iliac arteries. Description of the vascular access site with minimal luminal diameter, tortuosity, mural calcifications, location, and status of common femoral artery CFA (e.g., anterior luminal calcifications). Note: Optimal anatomical configurations for EVAR are indicated with letters, whereas suboptimal or unfavorable anatomical parameters are indicated within parentheses 
procedures during or after $\operatorname{EVAR}[15,16]$. In general, four anatomical areas thereby have to be addressed: (a) proximal fixation site, (b) aneurysm morphology, (c) distal fixation site, and (d) access vessel evaluation.

In general, optimal anatomical AAA configuration for EVAR includes an infrarenal aortic neck length of $>25 \mathrm{~mm}$; no mural neck thrombus/calcifications; no reverse taper infrarenal neck; lack of AAA tortuosity or angulation; patent non-tortuous, non-stenotic iliac arteries with a distal landing zone of $>30 \mathrm{~mm}$; patient internal iliac arteries; and normal common femoral artery non-calcified anatomy.

As with EVAR a suitable landing zone is of utmost importance for the success of the TEVAR procedure. A proximal landing zone of 15-25 mm is desirable [17]. Depending on the anatomic conditions, a sufficient proximal landing zone may need to be generated by supra-aortic debranching surgery, in which, for example, the left subclavian artery is transposed to the brachiocephalic trunk (Fig. 19.2). By doing so the left subclavian ostium can be overstented, and the proximal landing zone will be enlarged. Overstenting the left subclavian artery without prior debranching increases the risk of a type II endoleak, a subclavian steal phenomenon or ischemia of the vertebral artery territory, of the left upper extremity or spinal ischemia. Variants of the supra-aortic arteries must be taken into account when evaluating the proximal landing zone [9].

Thrombotic plaques in the aortic arch increase the risk of embolic stroke since catheter and stent-graft maneuvers in the arch may loosen the plaque [18]. Assessing the plaque burden of the thoracic aorta with focus on mobile atheromas is important to estimate the risk of embolic stroke which is as high in TEVAR as in open surgery with 4-10\% [6, 19].

The risk of spinal ischemia with paraplegia due to overstenting the artery of Adamkiewicz feeding intercostal artery, which is of variable location, is lower in TEVAR procedures than open surgery [20]. Extensive coverage of the thoracic aorta by the stent-graft and coverage of the left subclavian artery are recognized risks of spinal ischemia following TEVAR. Occurrence of stroke following TEVAR with left subclavian coverage is almost twice as high as following TEVAR without left subclavian coverage (6.3\% vs $3.2 \%)$ [21, 22].

Proper alignment of the stent graft to the aortic wall depends on the curvature of the aortic arch and on the conformability of the stent-graft material. In a sharp angulating aortic arch, the forces on the stent graft will hamper proper alignment, and the risk of a type I endoleak increases. Proper and in some cases adaptive radial forces of the stent-graft enable seamless adaption of the stent-graft to the inner and outer curvature of the aortic arch without leaving space for an endoleak and without damaging the aortic wall.

For better adaption to the aortic wall, tapered stent grafts are also available that have a conic architecture and adapt to the aortic wall despite different luminal diameters of the proximal and distal landing zones. Low-profile stent-graft systems enable better handling of the graft and access through smaller or via kinging iliac vessels.
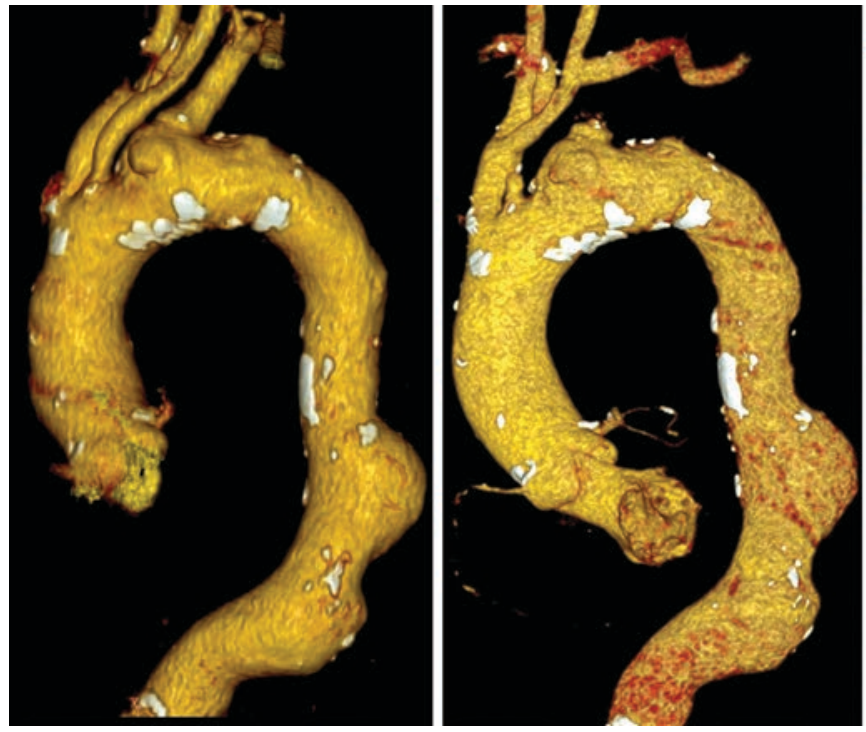

Fig. 19.2 Volume-rendered CT images of a 72-year-old lady with aneurysmal aortic disease readily reveal penetrating aortic ulcer of the aortic arch at the level of the ostium of the left subclavian artery and two sequential aneurysms of the descending thoracic aorta (a). Debranching
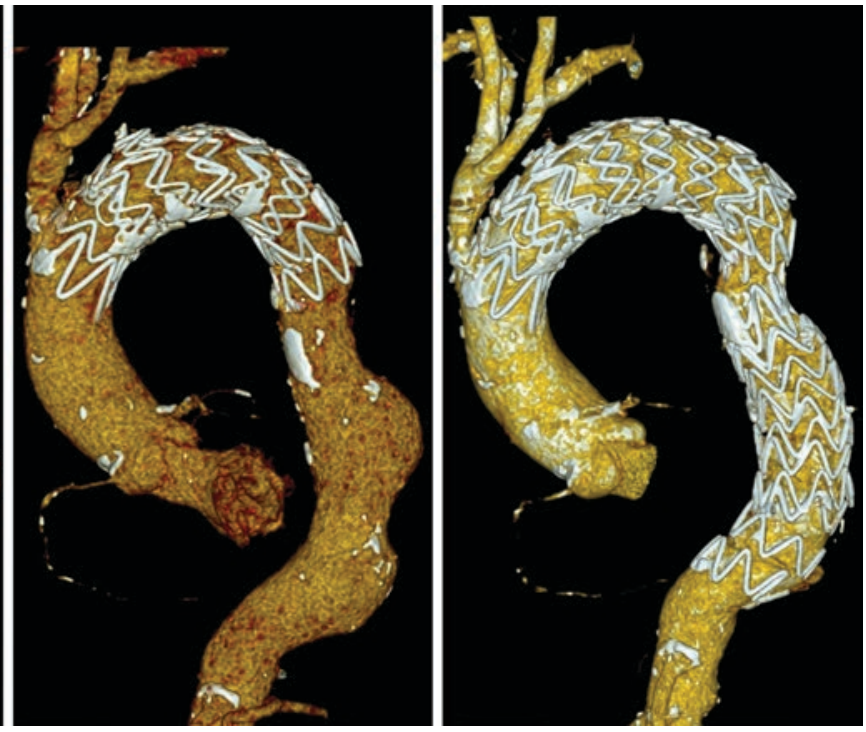

surgery with transposition of the left subclavian and left common carotid artery to the brachiocephalic trunk created a suitable proximal landing zone (b) for TEVAR placement (c). In the second step, the descending aortic aneurysms were treated with TEVAR extension (d) 


\section{Key Point}

- Every radiology pre-procedural imaging report has to address specific anatomical and morphological parameters allowing to accurately select the right EVAR device, to anticipate procedural challenges, and to plan for ancillary procedures during or after EVAR.

\subsection{Post-procedural Imaging}

Post-procedural surveillance imaging primarily focuses on early detection of EVAR stent-graft complications and failures. The complications may manifest immediately flowing EVAR, may resolve or persist over time, or appear at a time remote from the procedure itself.

The most common complication is the development of an endoleak following EVAR. With an endoleak, part of the blood flow remains outside the endograft and goes into the native aneurysmal sac. Endoleaks are classified by their source of blood flow and subclassified based on their location (Fig. 19.3). Approximately $25 \%$ of patients within 30 days post EVAR develop an endoleak; however, endoleaks can first occur years after EVAR continued surveillance imaging is required. Five types of endoleaks have been classified $[7,8]$.

Type I endoleak (Fig. 19.4) occurs due to an incomplete endograft seal at the proximal (type Ia) or distal (type Ib) fixation site or due to a leak around an iliac occludes plug in a patient with a aorto-uniiliac device and fem-fem crossover device [23]. With an endoleak type I, CTA detects contrast flow passing outside the fixation seal directly into the aneurysmal sac. Predisposing factors for type I endoleak are suboptimal infrarenal neck configurations (e.g., mural thrombus, reverse taper neck), neck dilatation from EVAR remodeling, complex chimney graft installations, or endograft migration to due insufficient endograft fixation (e.g., endograft fracture). Type I endoleaks are normally treated with reinforcement of the proximal/distal seal with placement of extender cuffs/stents.

Type II endoleak (Fig. 19.4) is the most common type of endoleaks occurring in patients post EVAR [24-26]. The incidence to develop an endoleak type II is approx. $25 \%$ per year [27]. They are fed by one (type IIa) or multiple (type IIb) collateral arterial pathways, such as lumbar arteries or inferior mesenteric artery (IMA). With an endoleak type II, CTA detects contrast flow within the aneurysmal sac, which can be traced back to an offending feeding/draining native vessel. Since approx. $40 \%$ of type II endoleaks spontaneously occlude and are often not associated with aneurysm sac enlargement in the first year, they do not require prompt treatment and are rather observed. In contrast, type II endoleaks occurring later than 1 year require often treatment since they are associated with aneurysm enlargement. Interventional vascular radiology offers various methods to halt blood flow of the type II feeder vessels.

Type III endoleak (Fig. 19.5) is rare and related to structural failure of the device. Endograft factures have been described in earlier generations of devices. Disconnection between the aortic body component and the uniiliac contralateral limb is the most common cause of a type III endoleak [28]. CTA may detect the endograft fracture site resulting into a direct contrast influx in the aneurysm sac [5, 29]. Treatment occurs normally promptly by placing an extender
Typ I

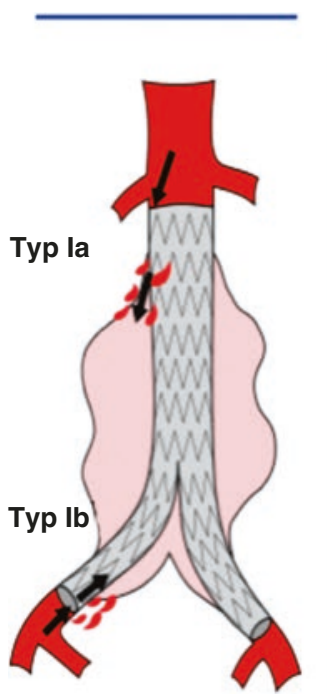

Typ II

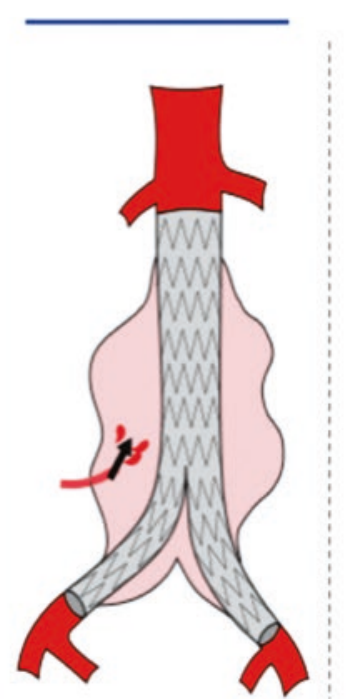

Typ III

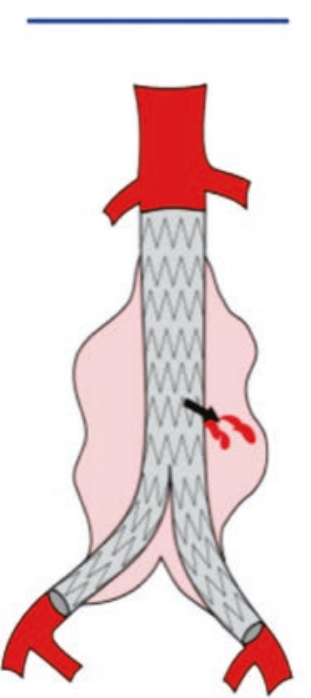

Typ IV

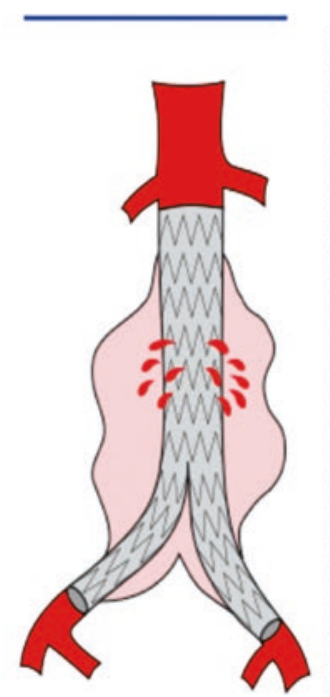

Typ V

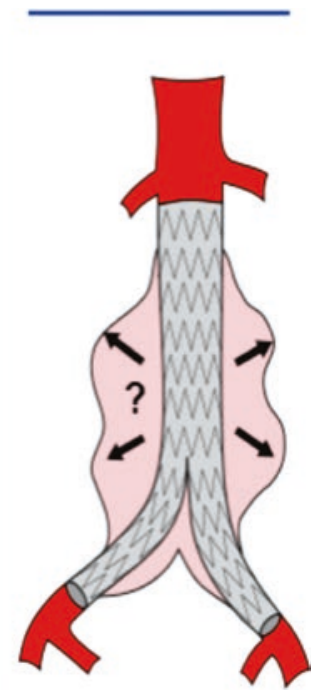

Fig. 19.3 Schematic drawing of the five types of endoleaks after aortic stent-graft placement 

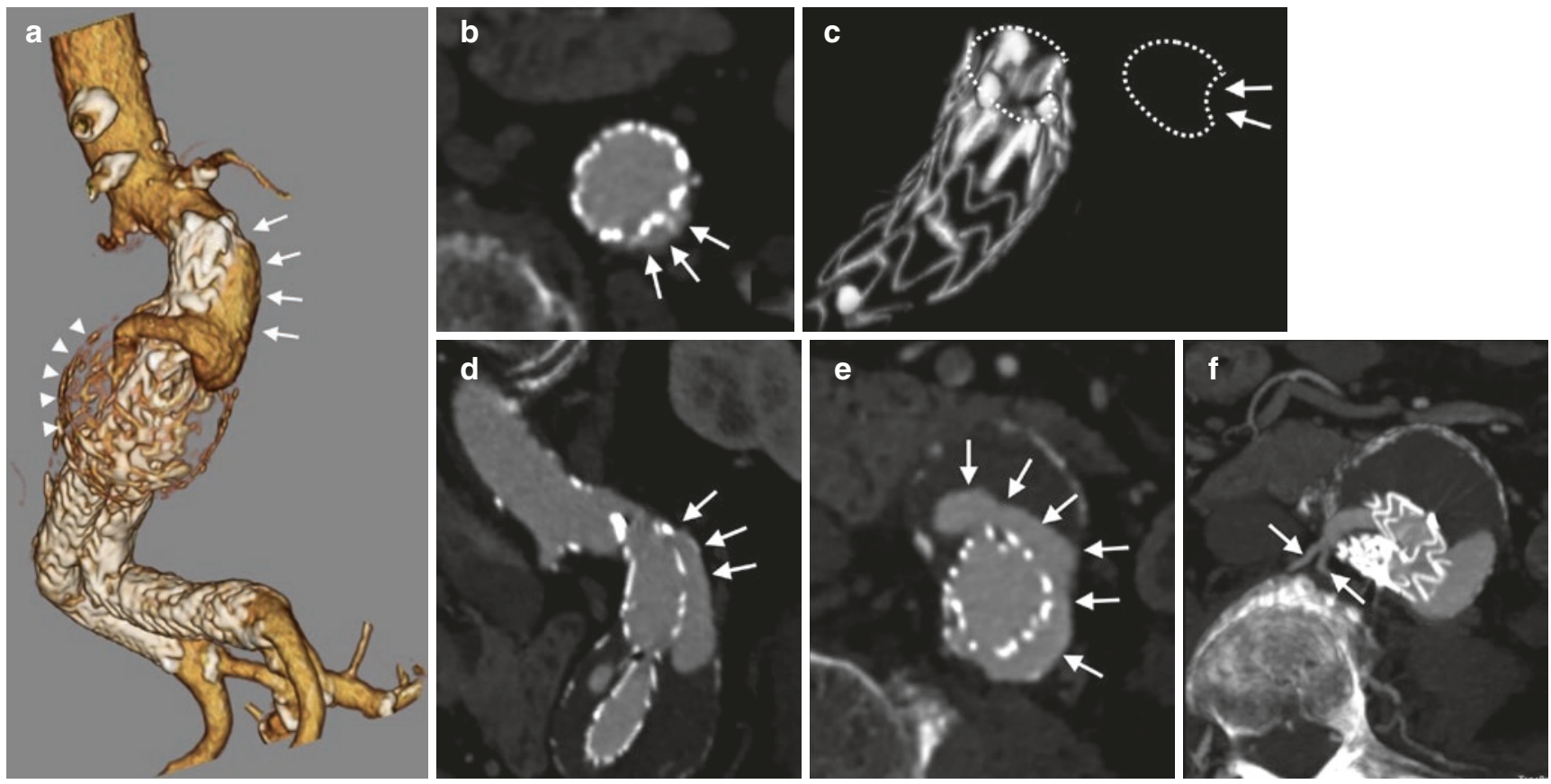

Fig. 19.4 Demonstration of type Ia and IIa endoleak in a patient 30 days post EVAR. (a) Volume-rendered image demonstrates contrast leaking along the proximal endograft fixation site (arrows) tracking into the aneurysm sac (aneurysm wall calcifications indicated by arrowheads). (b) Axial image at the uppermost proximal end of the endograft depicts contrast flowing outside the endograft lumen due to incomplete seal of the endograft device within the infrarenal neck. (c) Volume-

rendered image visualizes deformed stent-struts forming a channel for contrast and blood causing a type Ia endoleak (arrows). (d) Coronal reformatted image reveals the contrast column flowing outside and (e) around the endograft device within the aneurysm sac. (f) Axial thin slab MIP image demonstrates the communication of the type I endoleak with a type II endoleak fed by lumbar arteries
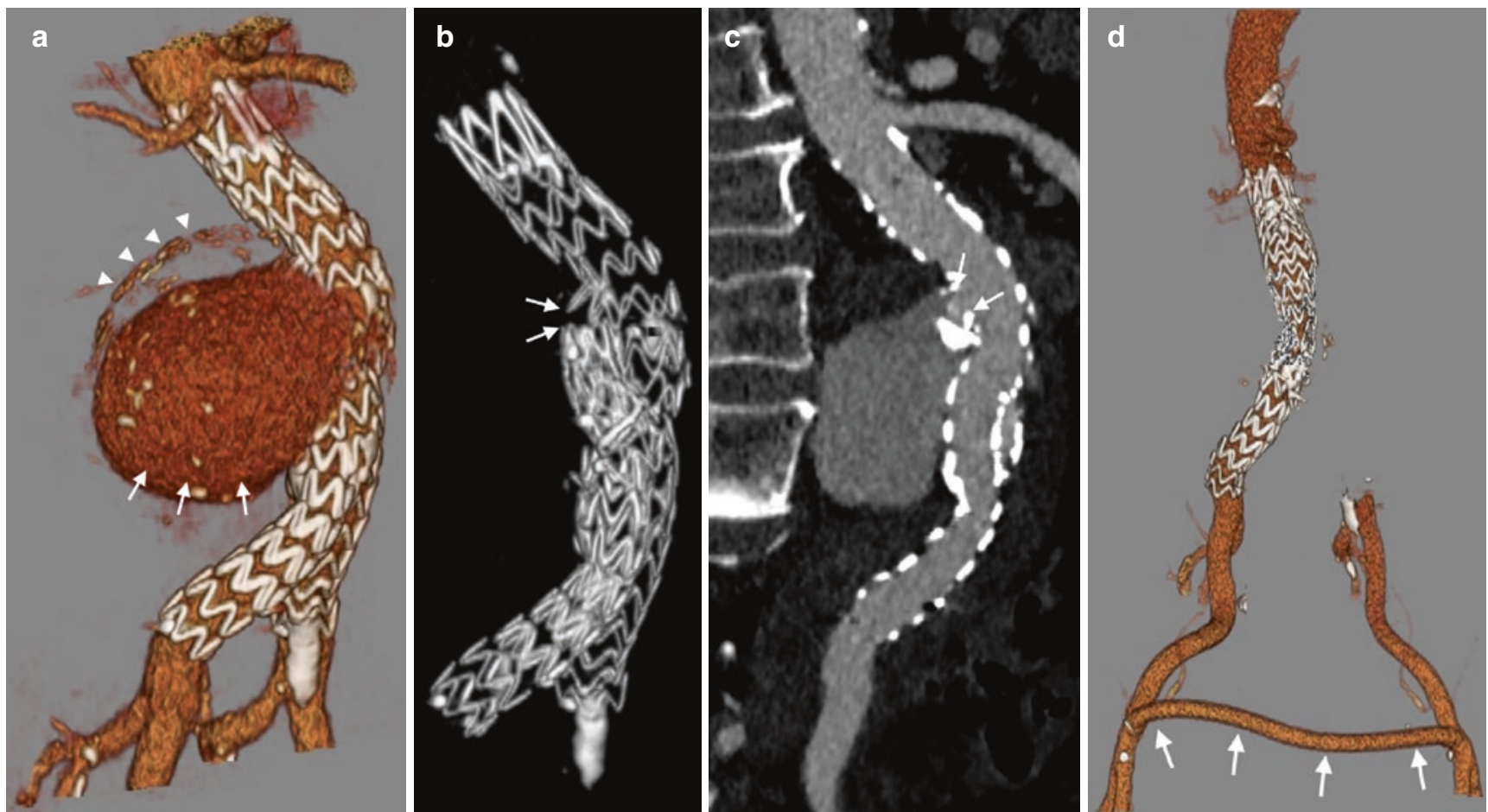

Fig. 19.5 Demonstration of type III endoleak in the first post-EVAR control. (a) Volume-rendered image demonstrates contrast leaking into the aneurysm sac (aneurysm wall calcifications indicated by arrowheads) at approx. Mid level of the endograft device. (b) Corresponding volume-rendered image reveals a stent-graft fracture at the proximal limb of the left uniiliac endograft limb (arrows). (c) Curved planar

reformatted image shows active contrast extravasation at the level of the structural failure (arrows) flowing into the aneurysmal sac. (d) Volumerendered image demonstrates result of immediate repair with placement of aorto-uniiliac endograft to seal the leak. Contralateral endograft limb is occluded; femoro-femoral bypass (arrows) provides blood flow to the pelvis and leg 
cuffs/stents. Since type III endoleaks may easily occur several years post EVAR, continuous surveillance is of paramount importance.

Type IV endoleak has never been seen with newergeneration devices. It is related to a transient graft fabric porosity, where contrast penetrating the fabric component can be seen as a blush with intra-arterial contrast injection. Type IV endoleak is typically of no clinical significance and rarely to never seen with CTA [30].

Type $\mathbf{V}$ endoleak, also called endotension, represents the group of growing aneurysms without any identifiable endoleak [31]. Endotension theories include systemic pressure transmittance across the graft to the aneurysm sac, present but not detectable type I-IV endoleaks, slow-grade infection, and difference in osmotic pressures among compartments [32]. Type $\mathrm{V}$ endoleaks normally occur later after EVAR, and surgical repair becomes necessary if aneurysm size becomes critical.

Other complications post EVAR include early complications at the vascular access site (thrombosis, dissection, pseudoaneurysm or AV fistula formation, infection), embolic mesenteric or renal disease related to migrated thrombotic material from the aneurysm sac, renal insufficiency, and endograft infection [33]. Endograft migration may occur several years after stent-graft placement (Fig. 19.6) [34].

Lifelong surveillance and meticulous attention to any possible endograft dysfunction are the goals to prevent the catastrophic event of a delayed rupture of EVAR. Mehta et al.
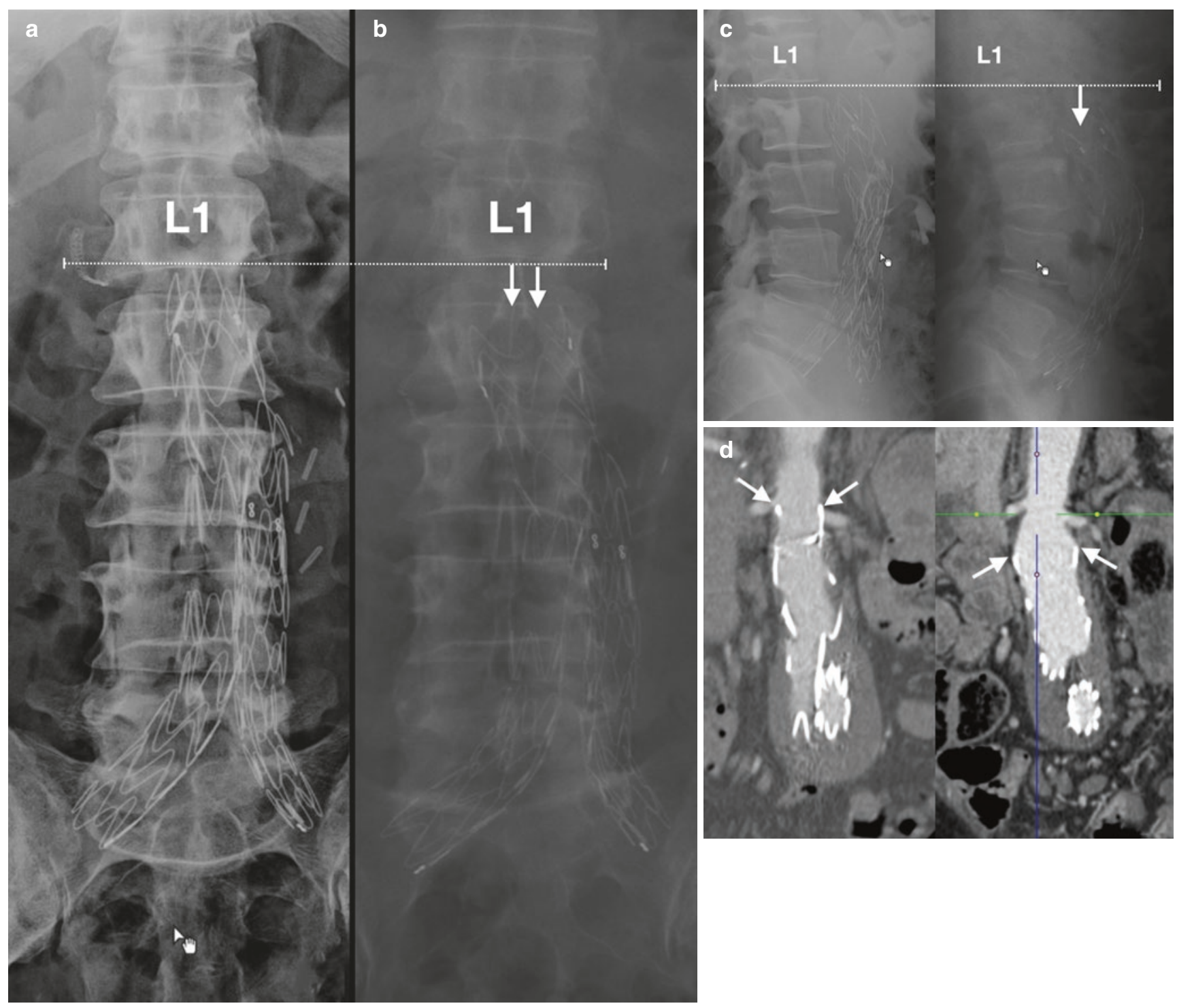

Fig. 19.6 Demonstration of slow endograft migration over a period of 6 years. (a) Abdominal ap plain film immediately post EVAR demonstrates endograft device in appropriate location with the proximal device end at the level of L1 lumbar inferior endplate. (b) Abdominal ap plain film demonstrates gradually inferior migration (arrows) of the endograft device. (c) Corresponding lateral views demonstrate significant inferior migration (arrow) of the endograft device. (d) Coronal multiplanar reformatted images confirm inferior migration of the proximal endograft device initially located at the orifice of the renal arteries (arrows) 
reported an incidence of delayed rupture after EVAR at $1.5 \%$ occurring at mean 29 months. The majority of patients had a type I endoleak (74\%), and endograft migration was present in $90 \%$ of patients [35]. Important to note is that patients with delayed ruptures may not show increasing aneurysm size on surveillance imaging. In case of rupture, unlike in native AAA rupture, the patients mostly remained hemodynamically stable and were able to undergo imaging. Emergent CTA imaging has to determine the underlying cause of rupture and to guide subsequent open surgical or endovascular repair.

\section{Key Point}

- Reliable detection of potential endoleaks, aneurysm sack volume increase, stent-graft migration, infection, and false aneurysm in the access vessel has to be assured for post-interventional surveillance of EVAR.

\subsection{Concluding Remarks}

CT angiography is the most valuable modality for pre- and post-imaging of aortic endovascular interventions. Assessment of the proximal and distal landing zones with meticulous attention to details of the branching vessels, aortic geometry, atheromatous plaques, and suitable access vessels are of utmost importance in the planning scan. Detection of potential endoleaks, aneurysm sack volume increase, stentgraft migration, infection, and false aneurysm in the access vessel are the main focus of post-intervention surveillance.

\section{Take-Home Messages}

- Aortic aneurysmal disease of the thoracic and abdominal aorta is a potentially life-threatening disease and requires besides preventive measures early detection of the disease and patient-tailored interventional treatment.

- Endovascular aneurysmal repair (EVAR) surpassed the method of open surgical repair since approximately a decade and became the method of choice to treat thoracic and abdominal aortic aneurysmal disease.

- Pre-procedural imaging reports have to address specific anatomical and morphological parameters in order to accurately select the right EVAR device, to anticipate procedural challenges, and to plan for ancillary procedures during or after intervention.
- In general, four anatomical areas have to be assessed: (a) proximal fixation site, (b) aneurysm morphology, (c) distal fixation site, and (d) access vessel evaluation.

- Post-interventional surveillance is of paramount importance in order to detect potential life-threatening early and late complications, such as stent-graft migration, endoleaks, aneurysm sack volume increase, delayed aortic rupture, or endograft infection.

\section{References}

1. Aggarwal S, Qamar A, Sharma V, et al. Abdominal aortic aneurysm: a comprehensive review. Exp Clin Cardiol. 2011;16:11-5.

2. Lederle FA, Johnson GR, Wilson SE, et al. Rupture rate of large abdominal aortic aneurysms in patients refusing or unfit for elective repair. JAMA. 2002;287:2968-72.

3. Ng TT, Mirocha J, Magner D, et al. Variations in the utilization of endovascular aneurysm repair reflect population risk factors and disease prevalence. J Vasc Surg. 2010;51:801-9, 809.e1.

4. Bryce Y, Rogoff P, Romanelli D, et al. Endovascular repair of abdominal aortic aneurysms: vascular anatomy, device selection, procedure, and procedure-specific complications. Radiographics. 2015;35:593-615.

5. Hallett RL, Ullery BW, Fleischmann D. Abdominal aortic aneurysms: pre- and post-procedural imaging. Abdom Radiol (NY). 2018;43:1044-66.

6. Riambau V, Böckler D, Brunkwall J, et al. Editor's choiceManagement of Descending Thoracic Aorta Diseases: clinical practice guidelines of the European Society for Vascular Surgery (ESVS). Eur J Vasc Endovasc Surg. 2017;53:4-52.

7. White GH, Yu W, May J. Endoleak-a proposed new terminology to describe incomplete aneurysm exclusion by an endoluminal graft. J Endovasc Surg. 1996;3:124-5.

8. Shah A, Stavropoulos SW. Imaging surveillance following endovascular aneurysm repair. Semin Interv Radiol. 2009;26:10-6.

9. Bean MJ, Johnson PT, Roseborough GS, et al. Thoracic aortic stent-grafts: utility of multidetector CT for pre- and postprocedure evaluation. Radiographics. 2008;28:1835-51.

10. Bavaria JE, Appoo JJ, Makaroun MS, et al. Endovascular stent grafting versus open surgical repair of descending thoracic aortic aneurysms in low-risk patients: a multicenter comparative trial. J Thorac Cardiovasc Surg. 2007;133:369-77.

11. Chan YC, Cheng SW, Ting AC, et al. Supra-aortic hybrid endovascular procedures for complex thoracic aortic disease: single center early to midterm results. J Vasc Surg. 2008;48:571-9.

12. Makaroun MS, Dillavou ED, Wheatley GH, et al. Five-year results of endovascular treatment with the Gore TAG device compared with open repair of thoracic aortic aneurysms. J Vasc Surg. 2008;47:912-8.

13. Matsumura JS, Melissano G, Cambria RP, et al. Five-year results of thoracic endovascular aortic repair with the zenith TX2. J Vasc Surg. 2014;60:1-10.

14. Maeda K, Ohki T, Kanaoka Y. Endovascular treatment of various aortic pathologies: review of the latest data and technologies. Int J Angiol. 2018;27:81-91. 
15. Chaikof EL, Fillinger MF, Matsumura JS, et al. Identifying and grading factors that modify the outcome of endovascular aortic aneurysm repair. J Vasc Surg. 2002;35:1061-6.

16. Picel AC, Kansal N. Essentials of endovascular abdominal aortic aneurysm repair imaging: preprocedural assessment. AJR Am J Roentgenol. 2014;203:W347-57.

17. Coady MA, Ikonomidis JS, Cheung AT, et al. Surgical management of descending thoracic aortic disease: open and endovascular approaches: a scientific statement from the American Heart Association. Circulation. 2010;121:2780-804.

18. Feezor RJ, Martin TD, Hess PJ, et al. Risk factors for perioperative stroke during thoracic endovascular aortic repairs (TEVAR). J Endovasc Ther. 2007;14:568-73.

19. Buth J, Harris PL, Hobo R, et al. Neurologic complications associated with endovascular repair of thoracic aortic pathology: incidence and risk factors. A study from the European collaborators on stent/graft techniques for aortic aneurysm repair (EUROSTAR) registry. J Vasc Surg. 2007;46:1103-10.. discussion 1110.

20. Cheng D, Martin J, Shennib H, et al. Endovascular aortic repair versus open surgical repair for descending thoracic aortic disease a systematic review and meta-analysis of comparative studies. J Am Coll Cardiol. 2010;55:986-1001.

21. Hajibandeh S, Antoniou SA, Torella F, et al. Meta-analysis of left subclavian artery coverage with and without revascularization in thoracic endovascular aortic repair. J Endovasc Ther. 2016;23:634-41.

22. Cooper DG, Walsh SR, Sadat U, et al. Neurological complications after left subclavian artery coverage during thoracic endovascular aortic repair: a systematic review and meta-analysis. J Vasc Surg. 2009;49:1594-601.

23. Stavropoulos SW, Clark TW, Carpenter JP, et al. Use of CT angiography to classify endoleaks after endovascular repair of abdominal aortic aneurysms. J Vasc Interv Radiol. 2005;16:663-7.

24. Arko FR, Rubin GD, Johnson BL, et al. Type-II endoleaks following endovascular AAA repair: preoperative predictors and longterm effects. J Endovasc Ther. 2001;8:503-10.
25. van Marrewijk CJ, Fransen G, Laheij RJ, et al. Is a type II endoleak after EVAR a harbinger of risk? Causes and outcome of open conversion and aneurysm rupture during follow-up. Eur J Vasc Endovasc Surg. 2004;27:128-37.

26. Jones JE, Atkins MD, Brewster DC, et al. Persistent type 2 endoleak after endovascular repair of abdominal aortic aneurysm is associated with adverse late outcomes. J Vasc Surg. 2007;46:1-8.

27. Veith FJ, Baum RA, Ohki T, et al. Nature and significance of endoleaks and endotension: summary of opinions expressed at an international conference. J Vasc Surg. 2002;35:1029-35.

28. Maleux G, Poorteman L, Laenen A, et al. Incidence, etiology, and management of type III endoleak after endovascular aortic repair. J Vasc Surg. 2017;66:1056-64.

29. Roos JE, Hellinger JC, Hallet R, et al. Detection of endograft fractures with multidetector row computed tomography. J Vasc Surg. 2005;42:1002-6.

30. Pandey N, Litt HI. Surveillance imaging following endovascular aneurysm repair. Semin Interv Radiol. 2015;32:239-48.

31. Ricotta JJ. Endoleak management and postoperative surveillance following endovascular repair of thoracic aortic aneurysms. J Vasc Surg. 2010;52:91S-9S.

32. Trocciola SM, Dayal R, Chaer RA, et al. The development of endotension is associated with increased transmission of pressure and serous components in porous expanded polytetrafluoroethylene stent-grafts: characterization using a canine model. J Vasc Surg. 2006;43:109-16.

33. Wilt TJ, Lederle FA, Macdonald R, et al. Comparison of endovascular and open surgical repairs for abdominal aortic aneurysm. Evid Rep Technol Assess (Full Rep). 2006:1-113.

34. Sampaio SM, Panneton JM, Mozes G, et al. AneuRx device migration: incidence, risk factors, and consequences. Ann Vasc Surg. 2005;19:178-85.

35. Mehta M, Paty PS, Roddy SP, et al. Treatment options for delayed AAA rupture following endovascular repair. J Vasc Surg. 2011;53:14-20.

Open Access This chapter is licensed under the terms of the Creative Commons Attribution 4.0 International License (http://creativecommons. $\mathrm{org} /$ licenses/by/4.0/), which permits use, sharing, adaptation, distribution and reproduction in any medium or format, as long as you give appropriate credit to the original author(s) and the source, provide a link to the Creative Commons license and indicate if changes were made.

The images or other third party material in this chapter are included in the chapter's Creative Commons license, unless indicated otherwise in a credit line to the material. If material is not included in the chapter's Creative Commons license and your intended use is not permitted by statutory regulation or exceeds the permitted use, you will need to obtain permission directly from the copyright holder. 